\title{
Abdominal pain with free peritoneal fluid detected by ultrasonography as a presenting manifestation of acute rheumatic fever
}

\author{
E Picard, A Gedalia, P Benmeir, N Zucker, Y Barki
}

\begin{abstract}
The clinical and laboratory data for an 8 year old girl with abdominal pain as a presenting manifestation of acute rheumatic fever are reported. An abdominal investigation with ultrasonography carried out at the same time showed free peritoneal fluid. These findings support the proposal that the abdominal pain classically described in acute rheumatic fever is one of the manifestations of the inflammatory process. It is suggested that in patients with abdominal pain, fever, and increased erythrocyte sedimentation rate in whom the diagnosis is not clear, an abdominal investigation with ultrasonography could be helpful in establishing the correct diagnosis.
\end{abstract}

Despite the decline in the occurrence of acute rheumatic fever in the USA during the last 20 years, there has been a resurgence of cases reported from different areas. ${ }^{1-6}$

In Israel, although acute rheumatic fever is less common than before, the disease is far from being completely eradicated. ${ }^{7}$ Abdominal pain has been known for many years as a manifestation of acute rheumatic fever. It is relatively uncommon, occurring in about $5 \%$ of patients, and usually precedes other rheumatic signs by a few hours or days. ${ }^{8} \mathrm{~A}$ possible sequence of events is that a patient with acute rheumatic fever may have acute abdominal pain and their normal appendix may be removed before the classical signs of the disease develop.

We report here a patient with acute rheumatic fever who initially presented with fever, diffuse abdominal pain, and free peritoneal fluid detected ultrasonographically. These findings support our proposal that, in acute rheumatic fever, abdominal pain is a consequence of the general inflammatory process.

\section{Case report}

An 8 year old girl was admitted to our department with a three day history of fever and diffuse abdominal pain without diarrhoea or vomiting. She had not had any previous episodes of acute rheumatic fever nor any recent sore throat or pharyngitis. Her parents were both Jews born in North Africa and there was no history of familial Mediterranean fever nor rheumatic diseases.

The patient presented as a well nourished girl with a temperature of $38^{\circ} \mathrm{C}$. On examination,

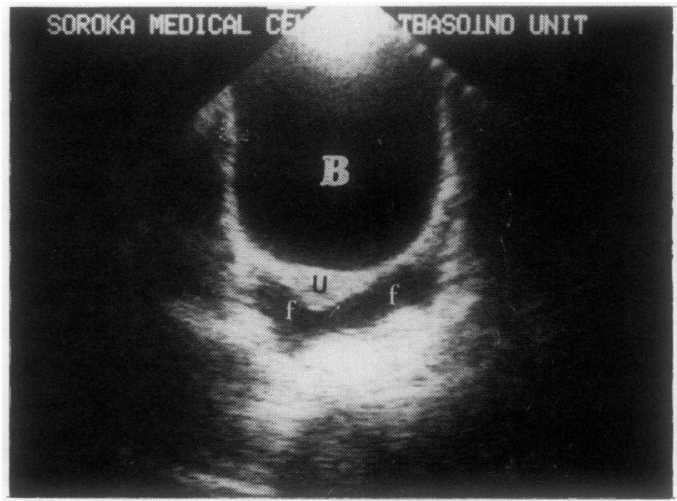

Transverse ultrasound view of the pelvis showing free peritoneal fluid $(f)$ behind the uterus $(u)$ and bladder $(B)$.

general abdominal tenderness with a defensive reaction was noted. No heart murmur was heard and there were no swollen joints. The rest of the physical examination was within normal limits. Laboratory studies showed the following values: haemoglobin $106 \mathrm{~g} / \mathrm{l}$; white blood cell count $17.6 \times 10^{9} / 1$ with $66 \%$ polymorphonuclear cells; erythrocyte sedimentation rate $100 \mathrm{~mm} / \mathrm{h}$; serum fibrinogen $9 \mathrm{~g} / \mathrm{l}$; antistreptolysin 0600 Todd units; antibodies to DNAase B 1/480. The patient was seen by a surgeon and appendicitis was suspected. An abdominal ultrasound examination was performed and showed free peritoneal fluid (figure). The patient received parenteral fluid and nothing by mouth and her condition improved. The abdominal symptoms disappeared gradually and on the fourth day after admission a regurgitative systolic murmur was heard at the apex radiating to the axilla. An echocardiographic examination showed a moderate mitral insufficiency with no left atrial enlargement. Two days later she developed pain in her right leg and a painful and swollen right ankle. Some hours later her right knee became red, swollen, and painful. A diagnosis of acute rheumatic fever with carditis was made. She was treated with an injection of benzathine penicillin and with aspirin.

The patient improved dramatically and within two days she was able to walk without pain. Two weeks later, the erythrocyte sedimentation rate was $35 \mathrm{~mm} / \mathrm{h}$ and the dose of aspirin was gradually decreased and stopped within the following week. She was discharged on a monthly regimen of benzathine penicillin injections. When examined after three, six and 12 months she remained well. 


\section{Discussion}

The patient reported in this paper presented with fever, abdominal pain, and increased erythrocyte sedimentation rate and white blood cell count. She then developed carditis and arthritis and had increased titres for antistreptolysin $\mathrm{O}$ and antibodies to DNAase. Based on the modified Jones criteria9 a diagnosis of acute rheumatic fever with carditis was made and the patient was treated accordingly. On admission, while the patient had fever and abdominal pain, the differential diagnosis included appendicitis and familial Mediterranean fever. Rheumatic abdominal pain can be difficult to distinguish from early acute appendicitis, particularly as the abdominal symptoms often precede other rheumatic manifestations. In acute rheumatic fever the abdominal pain is usually generalised and less likely to become localised to the right lower quadrant, as in acute appendicitis. The temperature is usually higher in acute rheumatic fever than in acute appendicitis. Although the white blood cell count is increased in both conditions, the erythrocyte sedimentation rate is often considerably increased in acute rheumatic fever and normal or only slightly increased in acute appendicitis. In some patients, when diagnosis remains in doubt, it is recommended to perform an operation. Acute abdominal pain is common in familial Mediterranean fever, especially among Jewish children of Sephardic origin, and has an autosomal recessive mode of inheritance. ${ }^{10}$ It is characterised by recurrent short episodes of fever amd polyserositis, usually peritonitis, arthritis, and pleuritis. Carditis is unlikely in an acute episode of familial Mediterranean fever.

The presence of abdominal pain as a nonspecific symptom of acute rheumatic fever has been described for a long time and is less common. ${ }^{11}$ Generally it is mild and disappears quickly, but can be severe and mimics appendicitis. $^{12}$ Davies has reported a case of a boy with abdominal pain manifested on each recurrence of rheumatic fever. ${ }^{13}$ Eight of 222 patients reported by Doliopoulos had an appendectomy during a relapse of acute rheumatic fever ${ }^{14} \mathrm{Lin}$ and Rodriguez-Torres have described five children in whom appendectomy was performed before any signs of acute rheumatic fever were present. ${ }^{15}$ Four of the five children had a normal appendix on laparotomy. We speculate that the free peritoneal fluid found during examination by ultrasonography in our patient with acute rheumatic fever represents peritonitis. The pathogenesis is probably an inflammatory and exudative process, as in the other organs in patients with acute rheumatic fever. This phenomenon is more common in the polyserositis of familial Mediterranean fever. The appearance of abdominal pain in acute rheumatic fever early in the onset of the disease is not understood. We suggest that in patients with acute abdominal pain with increased erythrocyte sedimentation rate, acute rheumatic fever should be included in the differential diagnosis. Examination of the abdomen by ultrasonography could be helpful in the confirmation of the diagnosis.

1 Land M A, Bisno A L. Acute rheumatic fever: a vanishing disease in suburbia. $7 A M A 1983 ; 249: 895-8$.

2 Markowitz M. The decline of rheumatic fever: role of medical intervention. F Pediatr 1985; 106: 545-50.

3 Veasy L G, Wiedmeier S E, Orsmond G S, et al. Resurgence of acute rheumatic fever in the intermountain area of the United States. N Engl f Med 1987; 316: 421-7.

4 Wald E R, Dashefsky B, Feldt C, Chiponis D, Byers C. Acute rheumatic fever in Western Pennsylvania and the tristate area. Pediatrics 1987; 80: 371-4.

5 Congeni B L, Rizzo C, Congeni J, Sreenivasan V V. Outbreak of acute rheumatic fever in Northeast Ohio. f Pediatr 1987; 144: 730-3.

6 Hosier D M, Craenen J M, Teske D W, Wheller J J. Resurgence of acute rheumatic fever. Am $\mathcal{F}$ Dis Child 1987; 144: $730-3$.

7 Bitton Y, Joseph A, Weinhouse E, Moses S. Review of 222 cases of acute rheumatic fever in Southern Israel. Pediatr Cardiol 1986; 7: 199-201.

8 Markowit: M, Gordis L. Rheumatic fever. 2nd ed. Philadelphia: Saunders, 1972: 77, 115.

9 Ad Hoc Committee to Revise the Jones Criteria (modified) Council on Rheumatic Fever and Congenital Heart Diseases of AHA. Jones criteria (revised) for guidance in the diagnosis of rheumatic fever. Circulation 1984; 69: 204A-8A.

10 McCarty D J. Arthritis and allied conditions. 11th ed. Philadelphia, London: Lea and Febiger, 1989: 995-6.

11 Giraldi J. Abdominal symptoms in acute rheumatism. Arch Dis Child 1930; 5: 379-81.

12 Keith J D, Rowe R D, Vlad P. Heart disease in infancy and childhood. 2nd ed. New York: Macmillan, 1967: 907

13 Davis E. Rheumatic fever: clinical, ecological and familial aspects. Springfield. IL: Charles C Thomas, 1969: 63, 91.

14 Doliopoulos T. Abdominal pain in rheumatic fever. Rheumatism 1951; 7: 42 .

15 Lin J S, Rodrigue\%-Torres R. Appendectomy in children with acute rheumatic fever. Pediatrics 1969; 43: 573-7. 